\title{
Multi-size, Multi-angle Microbipolar Forceps for Skull Base Surgery: Technical Note
}

\author{
Hamid Borghei-Razavi ${ }^{1} \quad$ Uta Schick ${ }^{1}$ \\ ${ }^{1}$ Department of Neurosurgery, Clemens Hospital, Münster, Germany \\ J Neurol Surg Rep 2015;76:e146-e150.
}

\begin{abstract}
Address for correspondence Hamid Borghei-Razavi, MD, Department of Neurosurgery, Clemens Hospital, Academic Hospital of Münster University, Düesbergweg 124, 48153 Münster, Germany

(e-mail: shbr61@yahoo.com; h.borghei-razavi@clemenshospital.de).
\end{abstract}

\begin{abstract}
Keywords

- bipolar forceps

- skull base surgery

- microsurgery

- hemostasis

- microdissection

Objective Hemorrhage control in skull base surgery is critical but hindered by the lack of instruments suitable for coagulating structural curves and corners. The main impediment is that most of the instruments currently used are right-angled and unsuitable because anatomical and pathologic structures are three-dimensional objects having complex curves and corners. In this article, we present a solution: the use of angled bipolar microforceps having a range of small diameters and angles for dissection and coagulation.

Methods Utilizing modern design software and up-to-date synthetic and metallic materials, a variety of nonstick bipolar microforceps with different angles and very fine tips $(0.2-1.2 \mathrm{~mm}$ ) were designed and constructed for use on different anatomical and pathologic curves. The tips of the forceps were made very fine to improve coagulation precision as well as to improve microdissection dexterity. The blades were made long and thin to improve visibility during coagulation and dissection procedures. As a result, these multi-size, multiangle micro instruments can be used not only for coagulation but also for microdissection or tumor removal in most anatomical areas accessed during the course of skull base surgery

Results The research, design, and construction of a new bipolar microforceps with different angles and sizes represents a technical innovation that can lead to improved surgical outcomes.

Conclusion The new micro-instruments enhance the quality and quantity of tumor and tissue resection and dissection in skull base surgery and open the possibility of new surgical approaches to microscopic tumor resection and hemorrhage coagulation in the anatomical areas of the skull base.
\end{abstract}

\section{Introduction}

The widespread use of nonstick microbipolar forceps for vascular coagulation and tissue dissection has led to a decrease in operating time and blood loss, thereby improving neurosurgical procedural outcomes.

Further improvements can be made because the current range of available microbipolar forceps does not

received

January 26, 2015

accepted

February 22, 2015

published online

June 1, 2015 adequately accommodate the complex shapes of many anatomical and pathologic structures. With this problem in mind, we developed a new, more adaptable type of nonstick microbipolar forceps with different angles and sizes.

In this article we introduce these instruments and highlight their technical details and clinical usefulness in the field of skull base surgery.
License terms Stuttgart · New York

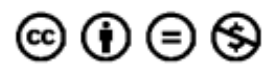




\section{Technical Description}

Making use of modern synthetic and metallic materials, these instruments were designed without the use of mechanical joints. All the movable elements are integrated within the instrument shaft, and despite the small diameter of the instruments, the blades can be opened smoothly to a width of $6 \mathrm{~mm}$.

The newly designed microforceps have an outer diameter of $1.9 \mathrm{~mm}$ and come in two different lengths: $200 \mathrm{~mm}$ and $230 \mathrm{~mm}$. Each of these lengths can have a 45-degree or an 80-degree microtip angled upward and downward (-Fig. 1) The new microforceps come in two microtip sizes: $2 \mathrm{~mm}$ for 45 degrees and $2.5 \mathrm{~mm}$ for 80 degrees (-Fig. 2A, B)

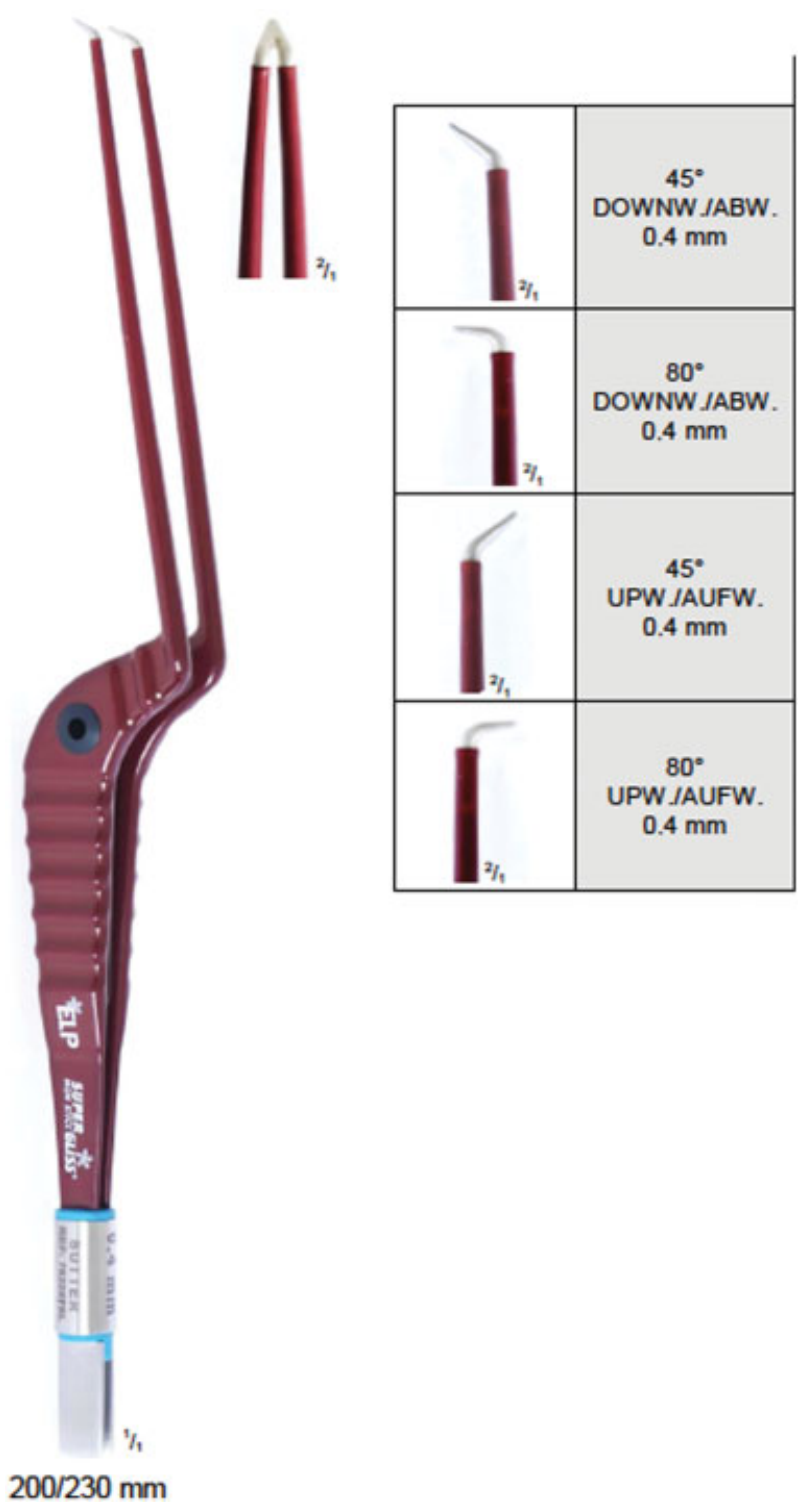

Fig. 1 The multi-angle microbipolar designed in two different lengths of $200 \mathrm{~mm}$ and $230 \mathrm{~mm}$. Each of the two lengths can have a 45- or an 80-degree angle upward and downward. UPW, upward; DOWNW, downward.
The sharp and fine microtip feature opens the possibility of microdissection, grasping and spreading out the tissues.

Depending on United States or European Union flat connector, the length of the bipolar forceps ranges between 190 and $220 \mathrm{~mm}$ and 200 and $230 \mathrm{~mm}$, respectively (-Fig. 3).

Each size microtip comes with a different colored flat connector so the surgeon and assistant can choose the correct tip size rapidly and accurately during the operation.

Engineering angled microbipolar forceps required a totally new approach that resulted in a completely new design that has been patented.

The microtips of the forceps are angled by elastic deformation of the metal without the use of any mechanical joints while retaining the same fineness for different microtip angles (45 and 80 degrees).

The tips of both standard and angled forceps are short and delicate to improve the precision of dissection of the arachnoid plane in particular. Moreover, the long and thin blades of the newly designed microforceps improve tissue visibility during coagulation, dissection, and tumor removal. The different dimensions of the angled micro-tip are shown in -Figs. 2A, B.

These improved version could be used in high-risk surgical situations such as optic canal, deep part of the internal auditory canal, and Meckel cave (see case presentation and discussion).

As previously stated, different types of flat connectors allow these new instruments to be used in different countries.

\section{Case Presentation}

A 55-year-old man presented with signs of increased intracranial pressure and left-sided scotoma as a result of a leftsided huge medial wing sphenoid meningioma with extension to the orbital, sellar, and ethmoidal regions as well as involvement of the cavernous sinus.

The location and size of the meningioma with consecutive optic nerve and optic canal involvement at the left side were identified by magnetic resonance imaging (MRI) (-Fig. 4A, B). The bony involvement of the anterior skull base and left optic canal and superior orbital fissure was identified by preoperative multi-slice skull base computed tomography scan.

The operation was performed via right-sided mini pterional craniotomy following extradural decompression of the anterolateral and posterolateral wall and roof of the orbit, and exposing the superior orbital fissure and foramen rotundom. ${ }^{1,2}$

Afterward, the dura was opened following dissection of the sylvian fissure and wide exposure of the tumor beside the carotid artery and optic nerve.

The previously described new angled microbipolar forceps were used intraoperatively for optimal access, easy coagulation, and even grasping and removal of the tumor around the optic nerve (-Fig. 5; - Video 1). During the procedure, the 


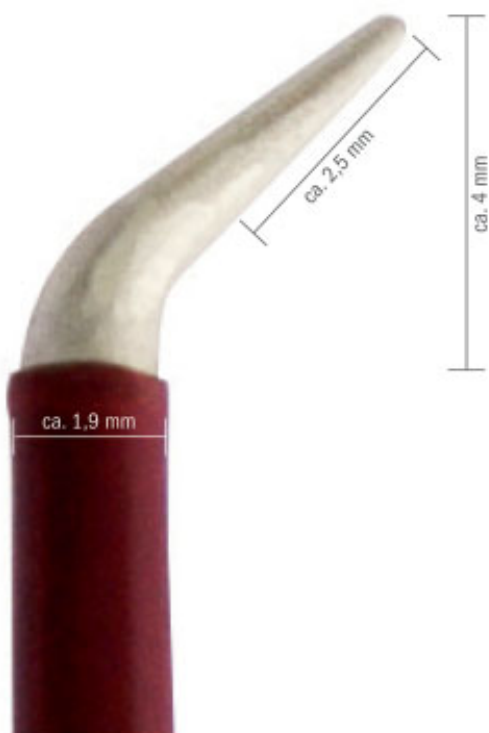

A

ELP Pinzette $45^{\circ}$ kurz aufwärts bzw. abwärts gewinkelt

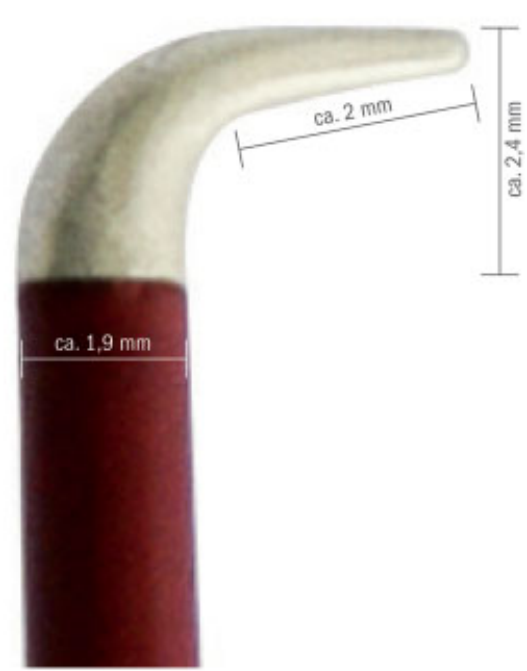

B

ELP Pinzette $80^{\circ}$ kurz aufwãrts bzw. abwärts gewinkelt

Fig. 2 Different length angled microtips. (A) 45 degrees. (B) 80 degrees.

surgeon did not have to change instruments frequently and was able to remove the tumor completely.

\section{Video 1}

Intraoperative use of angled microtip bipolar (45 degrees upward) in case described in Fig. 4. Online content including video sequence is viewable at: https://www.thieme-connect.com/products/ ejournals/html/10.1055/s-0035-1549313

The patient's postoperative clinical course was uneventful, with a total hospital stay of 8 days. A postoperative MRI taken 1 week after the operation revealed a total resection of the tumor, especially the extension of the tumor around the left optic nerve.

\section{Discussion}

Surgical access to lesions in the skull base, particularly access to lesions in the cerebellopontine angle and the petroclival region extending into Meckel cave and the posterior cavernous sinus, is a considerable surgical challenge. $^{3}$

These lesions were traditionally approached using a combination of supra- and infratentorial craniotomies. However, recent attempts have been made to avoid using these large combined approaches. Currently, to reduce the extent of surgical trauma, microsurgeons and endoscopic surgeons make an effort to avoid such approaches and try to minimize the size of the craniotomy as much as possible to reduce injury to the anatomical structures that must be disturbed to reach the tumor.
Nevertheless, these efforts continue to be hampered by the lack of development and availability of instruments suitable for microscopic and endoscopic skull base surgery.

A challenging area in skull base surgery is microsurgery of the tuberculum sella and spheno-orbital lesions. ${ }^{4,5}$ For instance, the complete surgical resection of a sphenoorbital meningioma is sometimes impossible because of the typical involvement of delicate structures of the orbital cone. The use of suitable micro instruments opens the possibility that the complete surgical resection of tumors such as meningioma in this anatomical region be the rule rather than the exception.

Another demanding area is the microsurgery of petroclival and jugular foramen meningioma. Depending on the origin, the meningioma can change the normal anatomy of the cranial nerves. ${ }^{6,7}$

In some remarkable cases, the tumor is completely anterior to the fifth to ninth cranial nerves, and the origin of the tumor is the anterior part of the jugular foramen. ${ }^{8}$ In such cases the microbipolar angled forceps are very helpful as a multipurpose instrument because the forceps can be used for cutting, coagulation, and grasping of the tumor ventral to and also between the fifth and ninth cranial nerves.

In our opinion, the new type of bipolar microforceps could also be used in operations of vestibular schwannoma via the retrosigmoid approach.

Most of the petroclival meningiomas arising medial to the trigeminal nerve (clival-type meningiomas) exhibit invasion into Meckel cave, with a high incidence (65\%) of adherence to the entry zone of the trigeminal nerve and around basilar perforators. $^{7}$

During the retrosigmoid intradural suprameatal approach to Meckel cave, it is possible to expose the anterior part of the trigeminal nerve and around the basilar perforators deeply using an angled endoscope. .,10 $^{9}$ 


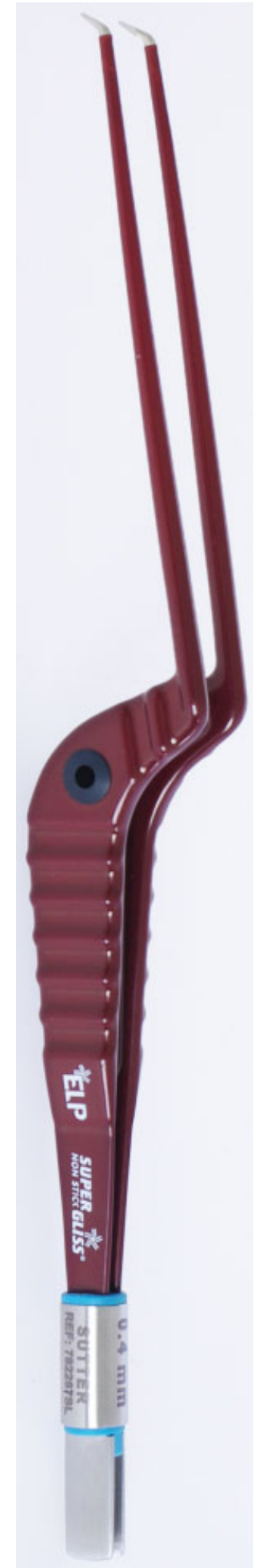

Fig. 3 The European model: 200 mm long, 45-degree angled microbipolar.
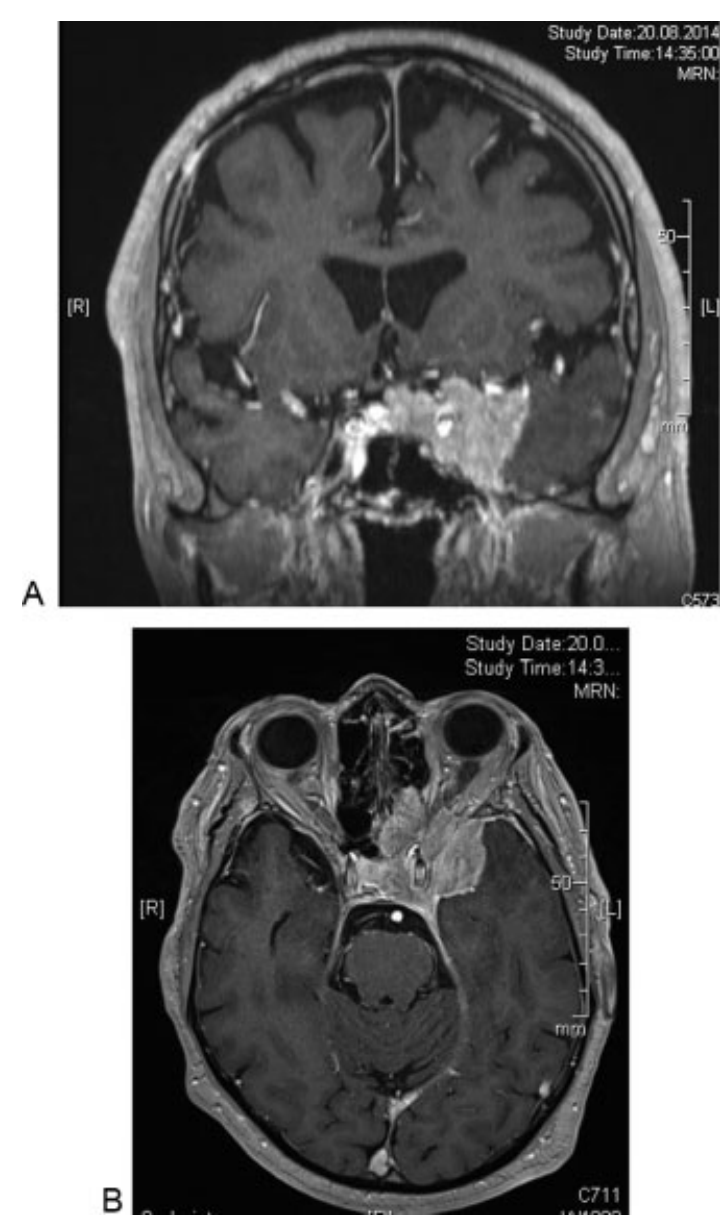

Fig. 4 Preoperative (A) coronal and (B) axial magnetic resonance imaging (with contrast) of a patient with left-sided medial sphenoid wing meningioma with extension to the orbital, sellar, and ethmoidal region as well as involvement of the cavernous sinus.

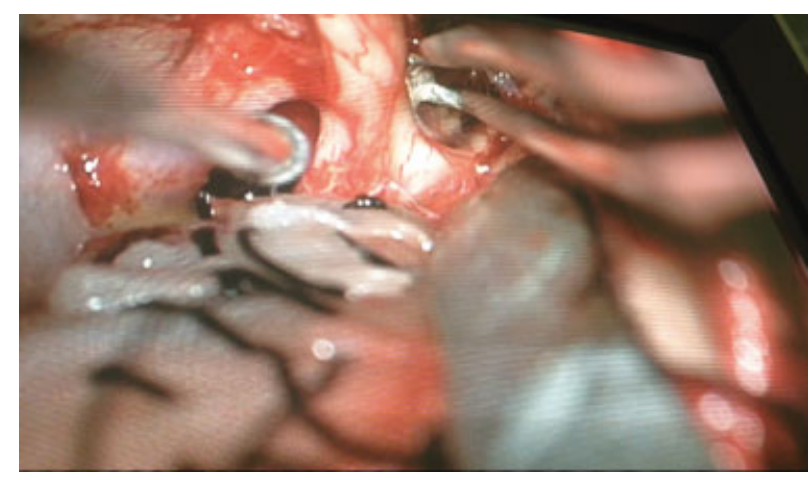

Fig. 5 Intraoperative use of angled microtip bipolar (45 degrees upward) in case described in Fig. 4 resulted in optimal access, easy coagulation, and even grasping and removal of the tumor around the left optic nerve (the suction tip shows left optic-carotid recess). 
In such approaches, more important than exposing the tumor area is the use of a suitable instrument for coagulation and dissection. We believe our newly developed microforceps are very suitable in this surgical approach.

The case presented here highlights the potential value of using multi-size multiangle microforceps in skull base surgery. The senior author has used these new instruments in $>100$ skull base operations and found them to be very effective for microdissection and coagulation of complex anatomical and pathologic structures.

\section{Disclosure}

The authors have no personal financial or institutional interest in any of the materials or devices described in this article. The authors are inventor of the instrument and it was produced and patented by Sutter Medizintechnik GmbH, Freiburg, Germany (Patent Number: US 7,513,897 B2).

\section{References}

1 Schick U. Meningiomas involving the sphenoid wing outcome after microsurgical treatment-a clinical review of 73 cases. Cent Eur Neurosurg 2010;71(4):198
2 Schick U, Hassler W. Decompression of endocrine orbitopathy via an extended extradural pterional approach. Acta Neurochir (Wien) 2005;147(2):143-149; discussion 149

3 Samii M, Tatagiba M, Carvalho GA. Retrosigmoid intradural suprameatal approach to Meckel's cave and the middle fossa: surgical technique and outcome. J Neurosurg 2000;92(2):235-241

4 Schick U, Hassler W. Surgical management of tuberculum sellae meningiomas: involvement of the optic canal and visual outcome. J Neurol Neurosurg Psychiatry 2005;76(7):977-983

5 Shrivastava RK, Sen C, Costantino PD, Della Rocca R. Sphenoorbital meningiomas: surgical limitations and lessons learned in their long-term management. J Neurosurg 2005;103(3):491-497

6 Safavi-Abbasi S, Zabramski JM, Deshmukh P, et al. Moving toward the petroclival region: a model for quantitative and anatomical analysis of tumor shift. J Neurosurg 2007;107(4):797-804

7 Ichimura S, Kawase T, Onozuka S, Yoshida K, Ohira T. Four subtypes of petroclival meningiomas: differences in symptoms and operative findings using the anterior transpetrosal approach. Acta Neurochir (Wien) 2008;150(7):637-645

8 Ramina R, Neto MC, Fernandes YB, Aguiar PH, de Meneses MS, Torres LF. Meningiomas of the jugular foramen. Neurosurg Rev 2006;29(1):55-60

9 Samii M, Tatagiba M, Carvalho GA. Resection of large petroclival meningiomas by the simple retrosigmoid route. J Clin Neurosci 1999;6(1):27-30

10 Komatsu F, Komatsu M, Di Ieva A, Tschabitscher M. Endoscopic approaches to the trigeminal nerve and clinical consideration for trigeminal schwannomas: a cadaveric study. J Neurosurg 2012; 117(4):690-696 